\title{
Synthesis of masked 2-amino-6-methyl-4-oxo-4H-pyran-3-carbaldehydes
}

\author{
Alexander V. Denisenko, ${ }^{\text {ab* }}$ Anton V. Tverdokhlebov ${ }^{\mathrm{b}}$ \\ ${ }^{a}$ Taras Shevchenko National University of Kyiv, Volodymyrska Street, 64/13, Kyiv 01601, Ukraine \\ ${ }^{\mathrm{b}}$ Enamine Ltd., Alexandra Matrosova str., 23, 01103 Kiev, Ukraine
}

\section{denisenkoalex@ukr.net}

Keywords: aminoaldehydes, nitriles, heterocycles, pyranes, hydrazones.

Acetoacetylation of (1,3-dimethylbenzimidazol-2-ylidene)-, (3-methylbenzothiazol-2-ylidene)-, and (3,4-dimethylthiazol-2-ylidene)acetonitriles with 2,2,6-trimethyl-4H-1,3-dioxin-4-one was found to yield appropriate $C$-acylation products. Treatment of the obtained products with perchloric acid afforded 2-(2-amino-6-methyl-4H-pyran-4-one-3-yl)substituted quaternary azolium salts. Their reduction with sodium borohydride yielded the corresponding dihydro (in the case of benzoazoles) or tetrahydro (in the case of thiazole) derivatives, which were shown to be synthetic equivalents of the title aldehyde.

\section{Introduction}

The $\quad N, N^{\prime}$-dimethylbenzimidazolium [1-5] and $N$-methylbenzothiazolium [6-9] moieties are well known as synthetic equivalents of aldehyde functionality. Their reduction into 2,3-dihydro derivatives yields the masked formyl group, which can, if necessary, be liberated by hydrolytic cleavage [1-9]. Recently, we successfully employed such an approach for the preparation of masked aldehydes of pyrrole $\mathbf{1}$ [10], fused pyrrole 2 [11], furan 3 [12] and pyridine 4 [13] series (Figure 1).

In continuation of our research on masked aldehydes we have extended our investigations to the study of 2-amino-6-methyl-4-oxo-4H-pyran3-carbaldehyde (5) derivatives. To the best of our knowledge, such compounds were not described to date.
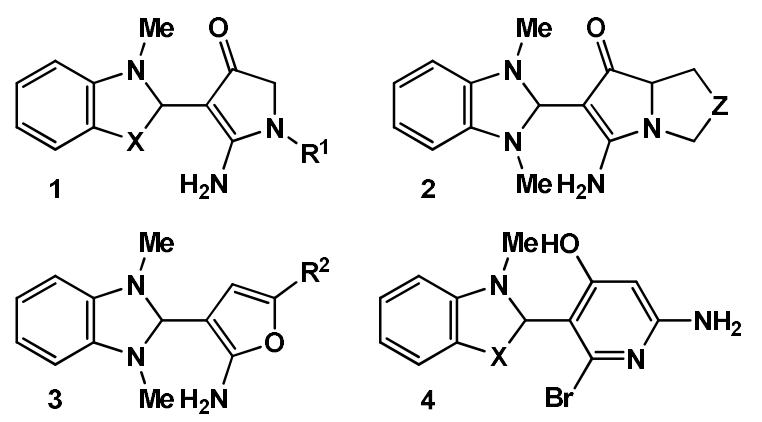<smiles>Cc1cc(=O)c(C=O)c(N)o1</smiles>

Figure 1. Previously prepared masked aldehydes. $X=\mathrm{NMe}$ or $\mathrm{S} ; \mathrm{R} 1=$ Alkyl or $\mathrm{Ar} ; \mathrm{R} 2=\mathrm{CF}_{3}$ or $\mathrm{Ar} ; \mathrm{Z}=\mathrm{CH} 2$ or $\mathrm{S}$.

\section{Results and Discussion}

Treatment of compounds 6a-c with 2,2,6trimethyl-4H-1,3-dioxin-4-one (7) as widely used agents for acetoacetylation [14-16] was found to yield $\mathrm{C}$-acylation products 8a-c 
(Scheme 1). It was found that reaction conditions strongly affect cyclization of the compounds 8ac. Thus, the use of $\mathrm{HBr} / \mathrm{AcOH}$ for the cyclization leads to formation of bromopyridines 9a-c, while the action of perchloric acid gives target aminopyranones 10a-c.

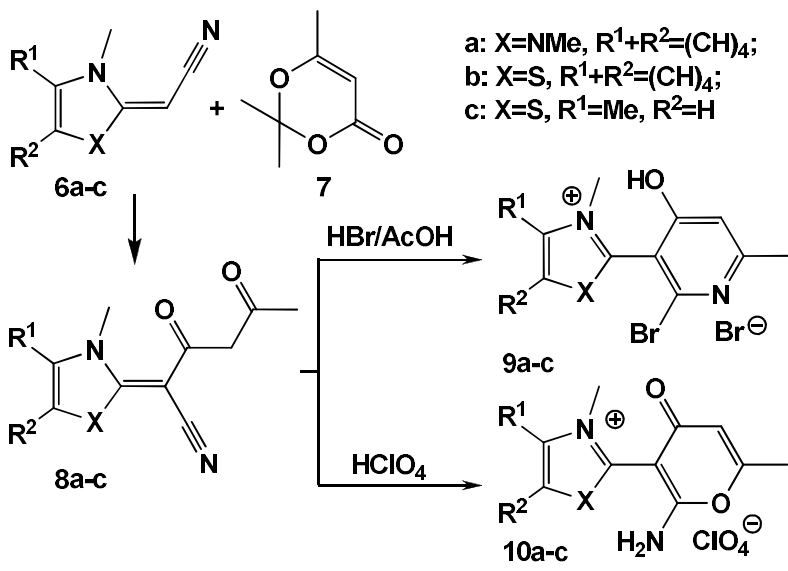

Scheme 1. Synthesis and reactions of derivatives 8a-c.

Probably the cyclization outcome is defined at the step of cation formation and depends on power of attacking nucleophile (Scheme 2).

Reduction of the quaternary salts $\mathbf{1 0 a}$ and 10b with excess sodium borohydride yielded the target masked 2-amino-6-methyl-4-oxo-4Hpyran-3-carbaldehyde derivatives 11a and 11b, respectively (Scheme 3 ). In the case of compound 10c, the reduction resulted in formation of the thiazolidine analogue 11c, which was similar to the previous results [13]. The structures of the obtained compounds 11a-c were confirmed by ${ }^{1} \mathrm{H}$ and ${ }^{13} \mathrm{C} \quad \mathrm{NMR}$ spectroscopic analysis (Scheme 3).

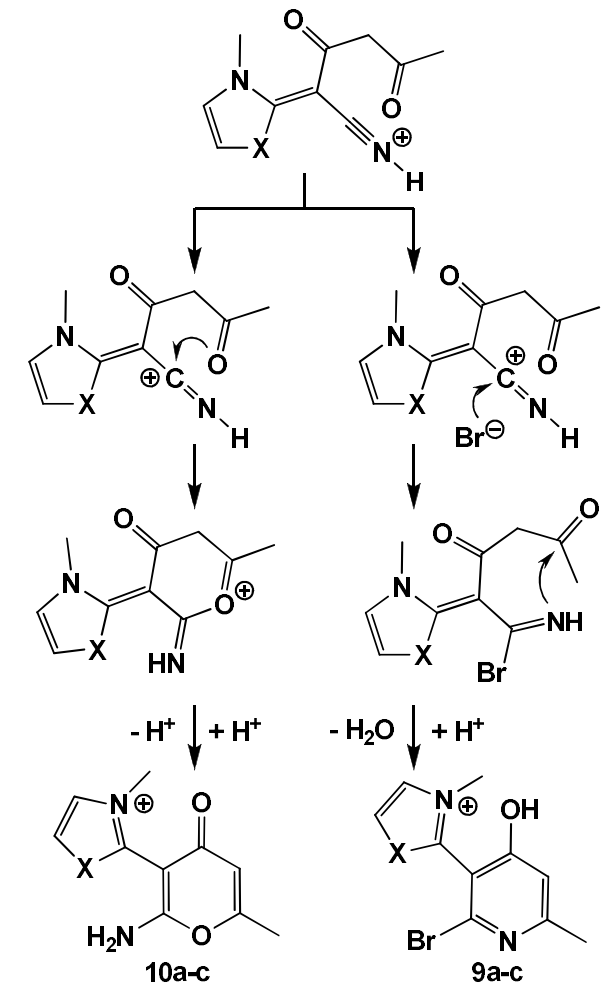

Scheme 2. Probable ways of formation of products 9 and 10.

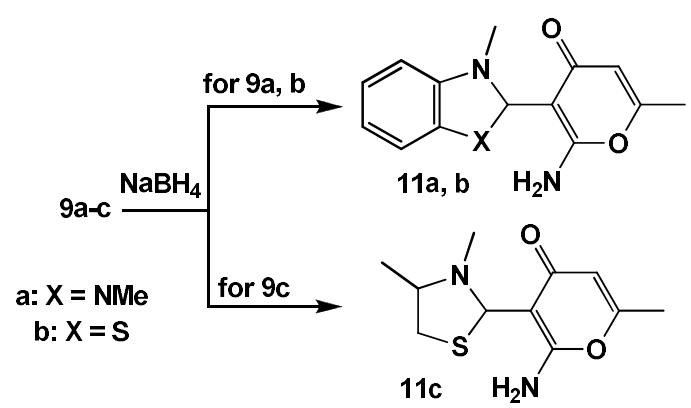

Scheme 3. Reduction of azolium salts 9a-c.

The aldehyde nature of compounds $\mathbf{1 1 a}-$ c was demonstrated by formation of the corresponding phenylhydrazone 12, semicarbazone 13 and 7-amino-2-methyl-4-oxo-4Hpyrano[2,3- $b]$ pyridine-6-carbonitrile $\mathbf{1 4}$ upon their treatment with phenylhydrazine, semicarbazide and malononitrile, respectively. Preparation of the same products 12-14 starting from all the derivatives $11 \mathbf{a}-\mathbf{c}$ is a good 
additional evidence for their structural assignments.

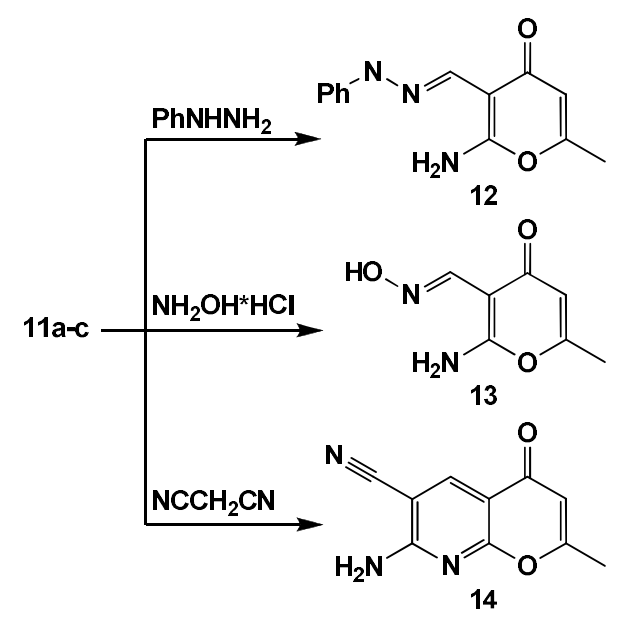

Scheme 4. Confirmation of aldehyde nature of derivatives $11 \mathbf{a}-\mathbf{c}$.

\section{Experimental part}

Material and methods

Nitriles 6a-c were prepared according to the described procedures [13]. Other reagents were commercially available. All melting points were determined in open capillary tubes with a Thiele apparatus and are uncorrected. ${ }^{1} \mathrm{H}$ and ${ }^{13} \mathrm{C}$ NMR spectra were recorded with a Bruker Avance 500 (500 MHz for ${ }^{1} \mathrm{H}$ and $125 \mathrm{MHz}$ for ${ }^{13} \mathrm{C}$ ) spectrometer in DMSO- $d_{6}$ solutions. Chemical shifts $(\delta)$ are given in ppm downfield from TMS as internal standard, $J$ values are in $\mathrm{Hz}$. The purities of all compounds were checked by ${ }^{1} \mathrm{H}$ NMR spectroscopic analysis and by LC/MS analysis on an Agilent 1100 instrument.

Synthesis

2-Heterylidene-3,5-dioxohexanenitriles 8a-c.

General Procedure. A solution of compound 6a-c $(50 \mathrm{mmol})$ and 2,2,6-trimethyl-4H-1,3dioxin-4-one (7) (9.24 g, $65 \mathrm{mmol})$ in anhydrous dioxane $(50 \mathrm{~mL})$ was heated at reflux for $4 \mathrm{~h}$. In the case of $\mathbf{6 a}$ reaction was performed without solvent at $120{ }^{\circ} \mathrm{C}$. After cooling, the reaction mixture was diluted with $i-\mathrm{PrOH}(80 \mathrm{~mL})$ and the precipitate formed was filtered, washed with $i$ $\mathrm{PrOH}$.

2-(1,3-Dimethyl-1,3-dihydro-2H-benzimidazol2-ylidene)-3,5-dioxohexanenitrile (8a). Beige solid. Yield 91\%, mp $89{ }^{\circ} \mathrm{C} .{ }^{1} \mathrm{H}$ NMR (mixture of keto/enol tautomers): $\delta=1.92$ and $2.23(2 \times \mathrm{s}$, $3 \mathrm{H}, \mathrm{CH}_{3}$ ), 3.71 (s, $1.4 \mathrm{H}, \mathrm{CH}_{2}$-keto), 3.76 and $3.78\left(2 \times \mathrm{s}, 6 \mathrm{H}, 2 \times \mathrm{NCH}_{3}\right), 5.69$ (br s, $0.3 \mathrm{H}, \mathrm{CH}-$ enol), 7.49-7.51 (m, 2 H, ArH), 7.77-7.79 (m, 2 $\mathrm{H}, \mathrm{ArH}) \mathrm{ppm} .{ }^{13} \mathrm{C}$ NMR: $\delta=22.1,30.8,33.3$, $33.5,54.8,60.7,95.5,112.3,121.2,122.5,125.3$, 125.4, 132.3, 132.4, 151.8, 152.2, 174.8, 183.4, 186.0, $204.2 \mathrm{ppm}$.

(2E)-2-(3-Methyl-1,3-benzothiazol-2(3H)-

ylidene)-3,5-dioxohexanenitrile (8b). Beige solid. Yield $86 \%$, mp $208^{\circ} \mathrm{C} . \delta=2.03$ and 2.24 (2×br s, $\left.3 \mathrm{H}, \mathrm{CH}_{3}\right), 3.92$ (s, $1.6 \mathrm{H}, \mathrm{CH}_{2}$-keto), 4.16 and $4.17\left(2 \times \mathrm{s}, 3 \mathrm{H}, \mathrm{NCH}_{3}\right), 5.94$ (br s, 0.2 $\mathrm{H}, \mathrm{CH}$-enol), 7.41-7.46 (m, $1 \mathrm{H}, \mathrm{ArH})$, 7.57-7.62 (m, 1 H, ArH), 7.73-7.77 (m, $1 \mathrm{H}$, ArH), 7.96-8.00 (m, $1 \mathrm{H}, \mathrm{ArH}) \mathrm{ppm} .{ }^{13} \mathrm{C}$ NMR: $\delta=22.1,30.9,36.3,36.6,54.7,77.8,96.2,113.5$, $119.6,120.5,122.8,123.1,125.1,125.2,126.7$, $127.3,127.9,128.0,140.3,140.5,165.2,165.5$, 177.2, 187.2, 187.5, $203.3 \mathrm{ppm}$. 


\section{(2E)-2-(3,4-Dimethyl-1,3-thiazol-2(3H)-}

ylidene)-3,5-dioxohexanenitrile (8c). Beige solid. Yield $88 \%$, mp $148{ }^{\circ} \mathrm{C} .{ }^{1} \mathrm{H}$ NMR (mixture of keto/enol tautomers): $\delta=1.96$ and $2.19(2 \times \mathrm{s}$, $\left.3 \mathrm{H}, \mathrm{CH}_{3}\right), 2.33$ (s, $\left.3 \mathrm{H}, \mathrm{CH}_{3}\right), 3.81(\mathrm{~s}, 1.5 \mathrm{H}$, $\mathrm{CH}_{2}$-keto), 3.90 (s, $3 \mathrm{H}, \mathrm{NCH}_{3}$ ), 5.79 (br s, 0.25 $\mathrm{H}, \mathrm{CH}-\mathrm{enol}), 7.01$ and $7.04(2 \times \mathrm{s}, 1 \mathrm{H}, \mathrm{ArH})$, 14.78 (s, $0.25 \mathrm{H}, \mathrm{OH})$ ppm. ${ }^{13} \mathrm{C}$ NMR: $\delta=14.4$, $14.5,30.9,36.7,37.0,54.4,72.8,76.0,95.8$, $108.7,109.5,120.2,121.3,140.3,140.5,164.5$, $1647,175.1,185.6,186.4,203.5 \mathrm{ppm}$.

\section{Quaternary Salts 9a-c. General Procedure.} Compound 8a-c (15 mmol) were dissolved in $40 \% \mathrm{HBr}$ in acetic acid $(30 \mathrm{~mL}$, obtained by saturation of acetic acid with gaseous $\mathrm{HBr}$ ) and the resulting solution was heated at reflux for 30 min. Upon cooling, the mixture was diluted with acetone $(50 \mathrm{~mL})$ and the solid precipitated was filtered, washed with cold acetone $(10 \mathrm{~mL})$.

\section{2-(2-Bromo-4-hydroxy-6-methylpyridin-3-yl)-}

1,3-dimethyl-1H-benzimidazol-3-ium bromide (9a). Beige solid. Yield $95 \%, \mathrm{mp}>250{ }^{\circ} \mathrm{C} .{ }^{1} \mathrm{H}$ NMR: $\delta=2.51\left(\mathrm{~s}, 3 \mathrm{H}, \mathrm{CH}_{3}\right), 3.95(\mathrm{~s}, 6 \mathrm{H}$, $\left.2 \times \mathrm{NCH}_{3}\right), 7.02(\mathrm{~s}, 1 \mathrm{H}, \mathrm{PyH}), 7.79-7.80(\mathrm{~m}, 2 \mathrm{H}$, ArH), 8.12-8.16 (m, $2 \mathrm{H}, \mathrm{ArH}) \mathrm{ppm} .{ }^{13} \mathrm{C}$ NMR: $\delta=24.0,33.0,105.0,105.9,112.1,114.3,114.4$, 127.8, 132.0, 141.1, 146.4, $167.4 \mathrm{ppm}$.

2-(2-Bromo-4-hydroxy-6-methylpyridin-3-yl)-3methyl-1,3-benzothiazol-3-ium bromide (9b).
Beige solid. Yield $76 \%$, mp $230{ }^{\circ} \mathrm{C}(\mathrm{dec}) .{ }^{1} \mathrm{H}$ NMR: $\delta=2.48\left(\mathrm{~s}, 3 \mathrm{H}, \mathrm{CH}_{3}\right), 4.23(\mathrm{~s}, 3 \mathrm{H}$, $\left.\mathrm{NCH}_{3}\right), 6.99$ (s, $\left.1 \mathrm{H}, \mathrm{PyH}\right), 7.93-7.96(\mathrm{~m}, 1 \mathrm{H}$, ArH), 8.00-8.04 (m, $1 \mathrm{H}, \mathrm{ArH}), 8.42-8.47$ (m, 1 $\mathrm{H}, \mathrm{ArH}), 8.66-8.70(\mathrm{~m}, 1 \mathrm{H}, \mathrm{ArH}) \mathrm{ppm} .{ }^{13} \mathrm{C}$ NMR: $\delta=23.7,38.1,110.1,112.2,112.8,115.2$, 118.6, 118.7, 122.7, 129.7, 129.8, 130.5, 130.6, 131.5, 131.6, 140.0, 141.4, 163.0, 166.8, 168.0, $169.6,169.7 \mathrm{ppm}$.

\section{2-(2-Bromo-4-hydroxy-6-methylpyridin-3-yl)-}

3,4-dimethyl-1,3-thiazol-3-ium bromide (9c). Beige solid. Yield $88 \%, \mathrm{mp}>250{ }^{\circ} \mathrm{C} .{ }^{1} \mathrm{H}$ NMR: $\delta=2.45\left(\mathrm{~s}, 3 \mathrm{H}, \mathrm{CH}_{3}\right), 2.65\left(\mathrm{~s}, 3 \mathrm{H}, \mathrm{CH}_{3}\right), 3.83$ (s, $\left.3 \mathrm{H}, \mathrm{NCH}_{3}\right), 6.90$ (s, $\left.1 \mathrm{H}, \mathrm{PyH}\right), 8.28(\mathrm{~s}, 1 \mathrm{H}$, ArH) ppm. ${ }^{13} \mathrm{C}$ NMR: $\delta=14.3,19.4,38.1,89.9$, 101.7, 123.2, 147.5, 147.3, 159.8, 161.9, 164.9 ppm.

\section{Quaternary Salts 10a-c. General Procedure.} Compound 8a-c (15 mmol) were dissolved in the mixture of $i-\mathrm{PrOH}(40 \mathrm{~mL})$ and $\mathrm{HClO}_{4}(60 \%, 20$ $\mathrm{mL}$ ) and the resulting solution was heated at reflux for $30 \mathrm{~min}$. Upon cooling, the mixture was diluted with acetone $(50 \mathrm{~mL})$ and the solid precipitated was filtered, washed with cold acetone $(10 \mathrm{~mL})$.

2-(2-Amino-6-methyl-4-oxo-4H-pyran-3-yl)-1,3dimethyl-1H-benzimidazol-3-ium perchlorate (10a). Beige solid. Yield $90 \%, \mathrm{mp}>250{ }^{\circ} \mathrm{C} .{ }^{1} \mathrm{H}$ NMR: $\delta=2.28\left(\mathrm{~s}, 3 \mathrm{H}, \mathrm{CH}_{3}\right), 3.86(\mathrm{~s}, 6 \mathrm{H}$, $\left.2 \times \mathrm{NCH}_{3}\right), 6.08(\mathrm{~s}, 1 \mathrm{H}, \mathrm{ArH}), 7.71$ (br s, $2 \mathrm{H}$, 
ArH), 8.06 (br s, 2 H, ArH), 8.28 (br s, $2 \mathrm{H}$, $2 \times \mathrm{NH})$ ppm. ${ }^{13} \mathrm{C}$ NMR: $\delta=19.3,32.6,82.4$, $110.3,113.7,126.7,132.6,146.8,162.6,164.5$, $175.7 \mathrm{ppm}$.

2-(2-Amino-6-methyl-4-oxo-4H-pyran-3-yl)-3methyl-1,3-benzothiazol-3-ium perchlorate (10b). Beige solid. Yield 83\%, mp $>250{ }^{\circ} \mathrm{C} .{ }^{1} \mathrm{H}$ NMR: $\delta=2.28\left(\mathrm{~s}, 3 \mathrm{H}, \mathrm{CH}_{3}\right), 4.07(\mathrm{~s}, 3 \mathrm{H}$, $\left.\mathrm{NCH}_{3}\right), 6.02$ (s, $\left.1 \mathrm{H}, \mathrm{ArH}\right), 7.78$ (t, $1 \mathrm{H}, \mathrm{J}=7.2$ $\mathrm{Hz}, \mathrm{ArH}), 7.86$ (t, $1 \mathrm{H}, \mathrm{J}=7.2 \mathrm{~Hz}, \operatorname{ArH}), 8.23$ (d, $1 \mathrm{H}, \mathrm{J}=7.2 \mathrm{~Hz}, \operatorname{ArH}), 8.37$ (d, 1 H, J = 7.2 Hz, ArH), $12.01(\mathrm{~s}, 1 \mathrm{H}, \mathrm{NH}) \mathrm{ppm} .{ }^{13} \mathrm{C}$ NMR: $\delta=$ 19.6, 96.7, 98.8, 117.3, 124.4, 128.4, 129.5, $130.2,141.2,153.7,161.5,169.3,169.7 \mathrm{ppm}$.

2-(2-Amino-6-methyl-4-oxo-4H-pyran-3-yl)-3,4dimethyl-1,3-thiazol-3-ium perchlorate (10c). Beige solid. Yield $90 \%, \mathrm{mp}>250{ }^{\circ} \mathrm{C}$. ${ }^{1} \mathrm{H}$ NMR: $\delta=2.22\left(\mathrm{~s}, 3 \mathrm{H}, \mathrm{CH}_{3}\right), 2.54\left(\mathrm{~s}, 3 \mathrm{H}, \mathrm{CH}_{3}\right), 3.73$ (s, $\left.3 \mathrm{H}, \mathrm{NCH}_{3}\right), 6.02$ (s, $\left.1 \mathrm{H}, \mathrm{ArH}\right), 8.03(\mathrm{~s}, 1 \mathrm{H}$, ArH), 8.14 (br s, $2 \mathrm{H}, 2 \times \mathrm{NH}$ ) ppm. ${ }^{13} \mathrm{C}$ NMR: $\delta$ $=14.5,19.2$, 37.9, 87.81, 110.0, 120.8, 146.5, 162.2, 163.5, $175.2 \mathrm{ppm}$.

Masked 2-Amino-6-methyl-4-oxo-4H-pyran3-carbaldehydes 11a-c. General Procedure. $\mathrm{NaBH}_{4}(0.76 \mathrm{~g}, 20 \mathrm{mmol})$ was added in portions to an ice-cooled and stirred solution of the salt $10 \mathrm{a}-\mathrm{c}(5 \mathrm{mmol})$ in aqueous $\mathrm{MeOH}(20 \mathrm{~mL}$; $\mathrm{MeOH}-\mathrm{H}_{2} \mathrm{O}$, 7:3). After the addition was complete, the mixture was stirred at $0-5^{\circ} \mathrm{C}$ for 1 h. The precipitate formed was filtered and washed with $\mathrm{H}_{2} \mathrm{O}$.

2-Amino-3-(1,3-dimethyl-2,3-dihydro-1Hbenzimidazol-2-yl)-6-methyl-4H-pyran-4-one (11a). Beige solid. Yield 68\%, mp 200-201 ${ }^{\circ} \mathrm{C}$. ${ }^{1} \mathrm{H}$ NMR: $\delta=2.15\left(\mathrm{~s}, 3 \mathrm{H}, \mathrm{CH}_{3}\right), 2.48(\mathrm{~s}, 6 \mathrm{H}$, $\left.2 \times \mathrm{NCH}_{3}\right), 5.29(\mathrm{~s}, 1 \mathrm{H}, \mathrm{CH}), 5.84(\mathrm{~s}, 1 \mathrm{H}, \mathrm{ArH})$, 6.47-6.49 (m, 2 H, ArH), 6.61-6.63 (m, $2 \mathrm{H}$, ArH), 7.05 (br s, $2 \mathrm{H}, \mathrm{NH}_{2}$ ) ppm. ${ }^{13} \mathrm{C} \mathrm{NMR}: \delta=$ 19.0, 33.8, 84.5, 92.3, 107.4, 111.2, 119.7, 142.9, $160.1,163.9,178.1 \mathrm{ppm}$.

2-Amino-6-methyl-3-(3-methyl-2,3-dihydro-1,3benzothiazol-2-yl)-4H-pyran-4-one (11b). Beige solid. Yield $65 \%, \mathrm{mp} 215{ }^{\circ} \mathrm{C}$ (dec). ${ }^{1} \mathrm{H}$ NMR: $\delta$ $=2.08\left(\mathrm{~s}, 3 \mathrm{H}, \mathrm{CH}_{3}\right), 2.61\left(\mathrm{~s}, 3 \mathrm{H}, \mathrm{NCH}_{3}\right), 5.66$ (s, $1 \mathrm{H}, \mathrm{CH}), 6.39$ (s, $1 \mathrm{H}, \mathrm{ArH}), 6.43$ (d, $1 \mathrm{H}, \mathrm{J}=$ $7.6 \mathrm{~Hz}, \mathrm{ArH}), 6.59$ (t, $1 \mathrm{H}, \mathrm{J}=7.6 \mathrm{~Hz}, \mathrm{ArH}), 6.91$ (t, $1 \mathrm{H}, \mathrm{J}=7.6 \mathrm{~Hz}, \operatorname{ArH}), 6.96$ (d, $1 \mathrm{H}, \mathrm{J}=7.6$ $\mathrm{Hz}, \mathrm{ArH}), 10.51$ (s, $1 \mathrm{H}, \mathrm{NH}), 11.19$ (s, 1 H, NH) ppm. ${ }^{13} \mathrm{C}$ NMR: $\delta=18.9,32.9,73.5,93.4,109.1$, $116.4,118.8,141.9,148.7,160.9,166.0,178.4$ ppm.

2-Amino-3-(3,4-dimethyl-1,3-thiazolidin-2-yl)6-methyl-4H-pyran-4-one (11c). Beige solid. Yield 59\%, mp $134-135{ }^{\circ} \mathrm{C} .{ }^{1} \mathrm{H}$ NMR: $\delta=1.23$ $\left(\mathrm{d}, J=4.2 \mathrm{~Hz}, 3 \mathrm{H}, \mathrm{CH}_{3}\right), 2.37\left(\mathrm{~s}, 3 \mathrm{H}, \mathrm{NCH}_{3}\right)$, 2.76-2.93 (m, $\left.2 \mathrm{H}, \mathrm{SCH}_{2}\right), 3.11-3.18(\mathrm{~m}, 1 \mathrm{H}$, $\mathrm{NCH}), 5.34$ (s, 1 H, SCHN), 5.83 (s, 1 H, ArH), 6.48 (br s, $2 \mathrm{H}, \mathrm{NH}_{2}$ ). ${ }^{13} \mathrm{C} \mathrm{NMR}: \delta=18.6,19.2$, 
36.4, 37.0, 64.1, 74.0, 93.6, 107.9, 141.3, 161.1, $166.2177 .8 \mathrm{ppm}$.

Phenylhydrazone 12 and Oxime 13. A solution of compound 11a-c $\quad(3.0 \quad \mathrm{mmol})$ and phenylhydrazine hydrochloride $(0.48 \mathrm{~g}, 3.3$ $\mathrm{mmol})$ or hydroxylamine hydrochloride $(0.23 \mathrm{~g}$, $3.3 \mathrm{mmol})$ in $i$-PrOH $(10 \mathrm{~mL})$ was heated at reflux under argon atmosphere for $2 \mathrm{~h}$. After cooling, the mixture was poured into $\mathrm{H}_{2} \mathrm{O}$ (30 $\mathrm{mL}$ ) and the solid that separated was filtered, washed with $\mathrm{H}_{2} \mathrm{O}(5 \mathrm{~mL})$.

\section{2-Amino-6-methyl-4-oxo-4H-pyran-3-}

carbaldehyde phenylhydrazone (12). Yields 73-80\%, mp $100{ }^{\circ} \mathrm{C} .{ }^{1} \mathrm{H}$ NMR: $\delta=2.20(\mathrm{~s}, 3 \mathrm{H}$, $\left.\mathrm{CH}_{3}\right), 5.88(\mathrm{~s}, 1 \mathrm{H}, \mathrm{ArH}), 6.70(\mathrm{t}, 1 \mathrm{H}, \mathrm{J}=7.6 \mathrm{~Hz}$, Ph), 6.84 (d, 2 H, J = 7.6 Hz, Ph), 7.20 (t, 2 H, J $=7.6 \mathrm{~Hz}, \mathrm{Ph}), 8.25(\mathrm{~s}, 1 \mathrm{H}, \mathrm{CH}), 8.46(\mathrm{~d}, 2 \mathrm{H}$, $\left.\mathrm{NH}_{2}\right), 10.00(\mathrm{~s}, 1 \mathrm{H}, \mathrm{NH}) \mathrm{ppm} .{ }^{13} \mathrm{C} \mathrm{NMR}: \delta=$ $19.2,94.8,110.7,111.8,118.5,129.6,135.6$, 145.9, 160.2, 162.0, $176.1 \mathrm{ppm}$.

\section{2-Amino-6-methyl-4-oxo-4H-pyran-3-}

carbaldehyde oxime (13). Yields 64-71\%, mp $100{ }^{\circ} \mathrm{C} .{ }^{1} \mathrm{H}$ NMR: $\delta=2.17\left(\mathrm{~s}, 3 \mathrm{H}, \mathrm{CH}_{3}\right), 5.86(\mathrm{~s}$, $1 \mathrm{H}, \mathrm{ArH}), 8.13$ (br s, $\left.2 \mathrm{H}, \mathrm{NH}_{2}\right), 8.23(\mathrm{~s}, 1 \mathrm{H}$, $\mathrm{CH}), 10.74$ (s, $1 \mathrm{H}, \mathrm{OH}) \mathrm{ppm} .{ }^{13} \mathrm{C}$ NMR: $\delta=19.1$, $92.3,110.6,144.5,160.5,162.6,176.1 \mathrm{ppm}$.

7-Amino-2-methyl-4-oxo-4H-pyrano[2,3-

b]pyridine-6-carbonitrile (14). A mixture of compound $\mathbf{6 a}-\mathbf{c}(3.0 \mathrm{mmol})$, malononitrile $(0.40$ $\mathrm{g}, 6.0 \mathrm{mmol})$ and $\mathrm{NH}_{4} \mathrm{Cl}(0.02 \mathrm{~g}, 0.3 \mathrm{mmol})$ in dioxane $(10 \mathrm{~mL})$ was heated at reflux for $4-5 \mathrm{~h}$. After cooling, the precipitate formed was filtered, washed with $\mathrm{H}_{2} \mathrm{O} \quad(5 \mathrm{~mL})$ and recrystallized from DMF. Yields $38-47 \%, \mathrm{mp}$ $100{ }^{\circ} \mathrm{C} .{ }^{1} \mathrm{H}$ NMR: $\delta=2.31\left(\mathrm{~s}, 3 \mathrm{H}, \mathrm{CH}_{3}\right), 6.13(\mathrm{~s}$, $1 \mathrm{H}, \mathrm{ArH}), 7.95$ (br s, $\left.2 \mathrm{H}, \mathrm{NH}_{2}\right), 8.43(\mathrm{~s}, 1 \mathrm{H}$, PyH) ppm. ${ }^{13} \mathrm{C}$ NMR: $\delta=20.2,90.8,108.3$, $110.5,116.0,143.6,161.1,163.0,166.0,176.0$ ppm.

\section{Conclusions}

To summarize, the present study has resulted in the first synthesis of masked 2-amino6-methyl-4-oxo-4H-pyran-3-carbaldehydes.

Aldehyde functionality has been brought into the $4 H$-pyran core in the form of a quaternary azolium salts. The synthetic potential of obtained unique masked aldehydes is not studied comprehensively to date and, therefore, further investigations in the field are in progress.

\section{References}

[1] Craig JC, Ekwuribe NN, Fu CC, Walker KAM. Synthesis. 1981; 4: 303-305.

[2] Ramos JM, Tarazi M, Wuest JD. J Org Chem. 1987; 52: 5437-5442.

[3] Katritzky AR, Aslan DC, Oniciu DC. Tetrahedron: Asymmetry. 1998; 9: 2245-2251.

[4] Lee I-SH, Jeoung EH, Kreevoy MM. J Am Chem Soc. 1997; 119: 2722-2728.

[5] Lee I-SH, Jeoung EH. J Org Chem. 1998; 63: $7275-7279$.

[6] Corey EJ, Boger DL. Tetrahedron Lett. 1978; 19: 5-8. 
[7] Corey EJ, Boger DL. Tetrahedron Lett. 1978; 19: 9-12.

[8] Corey EJ, Boger DL. Tetrahedron Lett. 1978; 19: $13-16$.

[9] Chikashita H, Itoh K. Heterocycles. 1985; 23: 295-300.

[10] Tverdokhlebov AV, Denisenko AV, Tolmachev AA, Volovenko YuM. Synthesis. 2007; 12: $1811-1818$.

[11] Tverdokhlebov AV, Denisenko AV, Tolmachev AA, Volovenko YuM, Shishkina SV, Shishkin OV. Synthesis. 2009; 8: 1265-1270.

[12] Denisenko AV, Tverdokhlebov AV, Tolmachev AA, Volovenko YuM, Shishkina SV, Shishkin OV. Synthesis. 2010; 6: 1009-1013.

[13] Denisenko AV, Tverdokhlebov AV, Tolmachev AA, Volovenko YuM. Synthesis. 2011; 2: 251-256.

[14] Clemens RJ, Hyatt JA. J Org Chem. 1985; 50(14): 2431-2435.

[15] Shaabani A, Seyyedhamzeh M, Ganji N, Ng SW. J Iran Chem Soc. 2014; 11: 481-487.

[16] Hari DP, Waser J. J Am Chem Soc. 2017; 139(25): 8420-8423. 